\title{
MONITORING SYSTEMS FOR PIPELINE SAFETY BASED ON FBG SENSORS
}

\author{
FERDINANDO FELLI1, CLAUDIO PARIS ${ }^{2,3}$, ANTONIO PAOLOZZI ${ }^{3,2}$, CRISTIAN VENDITTOZZI $^{4}$, \\ GIOVANNI DE LELLIS ${ }^{1} \&$ IROSHI ASANUMA ${ }^{5}$ \\ ${ }^{1}$ DICMA, Sapienza University of Rome, Italy, \\ ${ }^{2}$ Centro Fermi-Museo Storico della Fisica e Centro Studi e Ricerche "Enrico Fermi”, Italy, \\ ${ }^{3}$ School of Aerospace Engineering, Sapienza University of Rome, Italy, \\ ${ }^{4}$ FGA, Universidade de Brasilia, Brasil, \\ ${ }^{5}$ Chiba University, Japan
}

\begin{abstract}
Events like earthquakes, landslides, tsunami and other important occurrences, related to human activities could affect heavily the security of the pipeline constructions. Pipeline used for transportation of gas, petroleum and other hydrocarbons could become dangerous for three main problems that can occur during the operation process:1-Explosions related to malfunction of the system itself; 2-defects of the utilized material; 3-Robbery and sabotage operations. In order to prevent these events, the technology needs to step up in the mitigation direction, introducing new systems capable to understand rapidly what is happening in the territory. Those circumstances could constitute a serious and unexpected occurrence, that could afflict a big mass of people in relatively quick time and with catastrophic consequences. Our research group, during these years, has been studying various FBG monitoring systems, for preventing and monitoring among other things these criticalities. The main advantages in using those fibre optic based systems are: high corrosion resistance, no electromagnetic interference, multiplexing capabilities and no need of re-calibration during the years. This paper presents a review of the activities in Sapienza University performed by the Department DICMA and the School of Aerospace Engineering research teams for designing and testing FBG-based vibration sensors that can be used for detecting damaging or illegal activities on pipelines and also for structural monitoring of high pressure pipes.
\end{abstract}

Keywords: FBG sensors, pipelines, disaster mitigation, research review.

\section{INTRODUCTION}

The increase in global energy demand has led to an increase in the installation of infrastructures for energy production and distribution in the territory. The installation of these mechanical and thermal systems is subjected to constant and various types of stress: vibrations caused by the city modernization works, natural phenomena, sabotages. All these types of stresses could lead to an increasing of pipeline networks critical issues; the consequences are, in the worst cases, the replacement of the entire line or a change of the materials used for the construction. Any damage to a pipeline, used for the distribution of gas, oil or other materials, could lead to a significant environmental pollution and loss of human lives. A risk that is underestimated, is the illegal spilling of hydrocarbons: that caused a certain number of accidents in the last few years (see for instance [1]. The pipeline theft, in most cases, is realized using illegal taps created on pipeline side and connecting these ones to hoses that bring the stolen oil to tanks or trucks used for the transportation [2]. During the spill, because of the holes created, an uncontrolled leaking of fuel can happen [3]; perpetuated over time, the risk of pollution caused by the oil increase significantly. Moreover, drilling pipes requires heavy vehicles, such as, excavators, cranes and drills; the vibrations caused by the vehicles could lead themselves to a failure due to the uncontrolled vibrations generated. On the other hand, the detector of unexpected vibrations could be a useful tool for detecting dangerous or illegal activities nearby a pipeline. 
The oil industry infrastructure seems insufficiently monitored because, over the years, theft is increasing, but traditional methods of monitoring seem to be not effective for stopping these thefts. The material flow variations, inside the buried pipes, it is not easy to detect by traditional devices, such as systems for volumetric variations; Surface inspections are not effective, because of the cost of the thermal cameras. For vibrations monitoring usually sensors such as accelerometers are used; the limitation in using these systems is the impossibility of multiplexing them, thus needing large numbers of cables and complex networks of monitoring stations. The use of electromechanical sensors is not convenient because of the harsh conditions of the pipeline operating environment. FBG sensors on the other hand have many advantages such as immunity from electromagnetic interferences that make them ideal for instance in high energy physics experiments [4]-[7]. They can be used as temperature sensors even in cryogenic environment [8], [9]. It is also relatively easy to embed them into materials both for structural analysis [10] and other particular studies [11]. Fibre optics are made of insulation material and so they are particularly interested in outdoor applications where electric discharge can occur during thunderstorms. This property allows to embed them even in metallic materials [12], [13]. Depending on the fibre optic interrogation system, they can be used for dynamic measurements [14], without frequency limitation, from the optical fibre point of view. Several types of sensing capabilities can be exploited from FBGs In Ref. [15], for instance among other things it has been proposed a new application of FBG sensor, namely a "Sand Load Cell" (SLC) that allow the measurement of the pressure due to the stratification of sand. This type of hazard is relevant for instance for railways located in desert areas. Before leaving this section, we would like to mention another advantage offered by the FBG sensors with respect to the conventional ones: multiplexing many sensors on the same fibre allows.to greatly simplify the network architecture.

\section{EXPERIMENTAL ACTIVITIES}

In order to keep under control, the pipeline parameters, we realized a device exploiting FBG sensors features. To amplify and detect the vibrations, instead of simply bonding the FBG sensor on the pipe, a special interface, named SPFD (Smart Pipeline FBG Device), has been designed and tested as described in the following paragraphs.

\subsection{Preliminary indoor test setup.}

The first experimental set-up, shown in Fig. 1, consists of a support where a steel pipe of length of about $0.6 \mathrm{~m}$, has been constrained at the half of its length leaving about $0.25 \mathrm{~m}$ of free pipe on each end. Four blind holes have been prepared to guide the drill in specific points along the pipe. The system itself is buried in sand to simulate piece of a pipeline buried below the ground and to add the damping effect of the ground. To compensate the temperature effect, a second FBG sensor has been positioned on the surface. The test has been performed using a drill with an overweight of 5 or $10 \mathrm{~kg}$, in order to simulate the pressure of the drill itself. The results have shown surprisingly that the higher the distance the larger the response (table 1). This can be explained by the fact that at short distances an amplification can occur due to bending of the pipe. In other words, being further away from the clamp will provide higher deflections that ultimately give a higher strain at the FBG location [16]. Besides identifying the drilling operation, the SPFD sensor is capable of detecting long term actions on the pipe such as the removal of the sand from the pipe. 


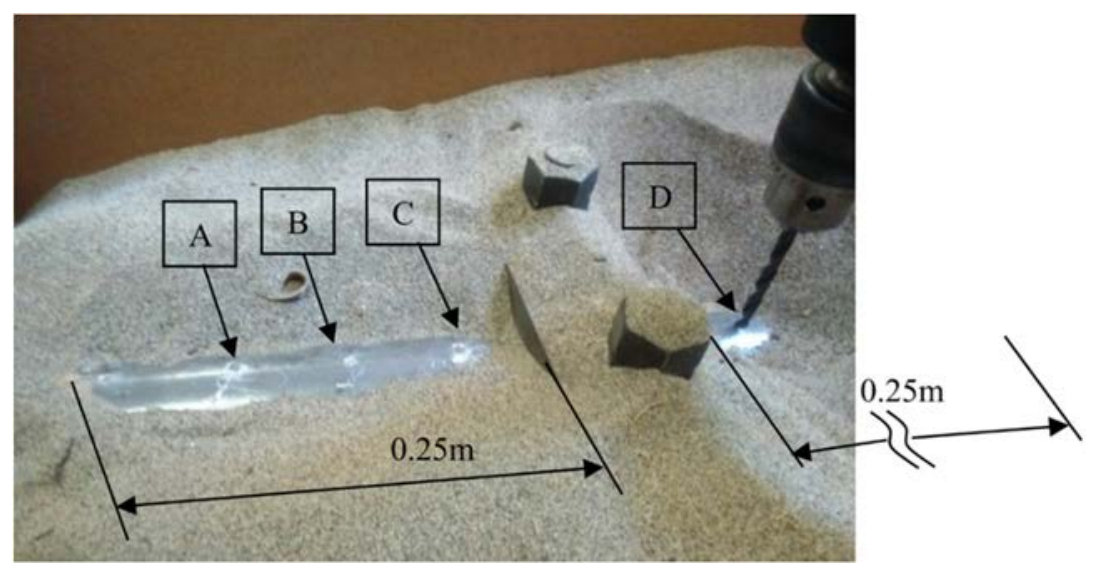

Figure 1: Laboratory set-up of a pipe buried with sand. On the right, the drill.

Table 1: Measured wavelength shifts on a $0.6 \mathrm{~m}$ buried pipe due to drilling.

\begin{tabular}{|l|c|c|}
\hline & Distance $^{1}[\mathrm{~cm}]$ & Max wavelength shift with $10 \mathrm{Kg}[\mathrm{nm}]$ \\
\hline A & 42 & 0.098 \\
\hline B & 34 & 0.044 \\
\hline C & 26 & 0.019 \\
\hline D & 16 & 0.012 \\
\hline \multicolumn{3}{|c|}{} \\
\hline
\end{tabular}

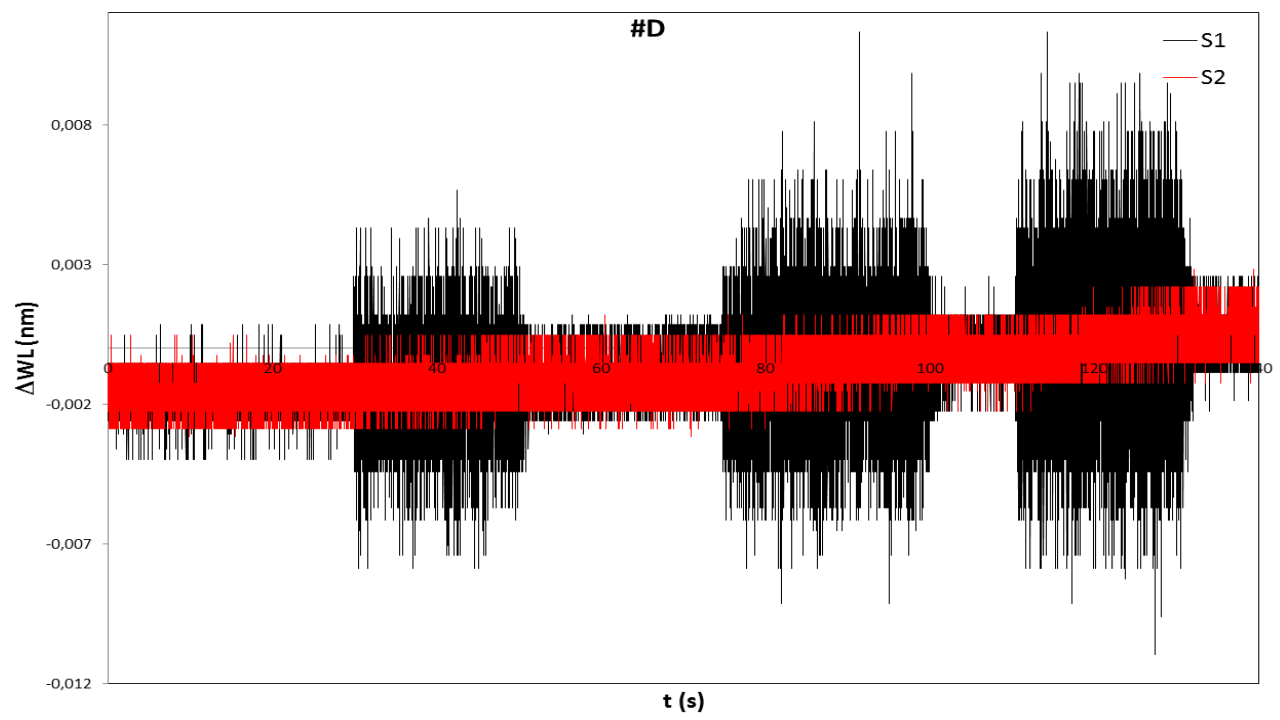

Figure 2: Wavelength shift of SPFD sensor (black line) and of FBG sensor (red line) while drilling and removing sand from the pipe. 
This can be seen in Fig. 2 where both the response measured by the SPFD reported in black (S1 line) and the FBG temperature sensor reported in red (S2 line). The black line clearly shows the increase in strain during the three phases of drilling operation, but also the slow increase in wavelength due to the increase of temperature, confirmed also by the behaviour of the red line.

\subsection{Outdoor test setup}

Following the promising results of the indoor tests, the SPFD vibration sensor has been tested on a longer pipe in an outdoor environment at the School of Aerospace Engineering of Sapienza University of Rome. The pipeline was part of the decommissioned hypersonic wind tunnel of the Progetto San Marco and the School.

Two blind holes were drilled on the pipe, one at $150 \mathrm{~cm}$ distance from the vibration sensor and $25 \mathrm{~cm}$ from the support number 2; the second hole was cut at $670 \mathrm{~cm}$ from the sensor with three supports between the sensor and the drill (Fig. 3).

Also, an FBG sensor for temperature compensation was fixed to the pipe. This sensor was also used for comparing the sensitivity of the vibration sensor with the sensitivity of a bonded FBG. The results of Table 1 are not confirmed at the longer distances used in this second test. In fact [17], the response in frequency domain at $150 \mathrm{~Hz}$ is a factor of 2.12 higher at $150 \mathrm{~cm}$ distance with respect to the response obtained at a distance of $650 \mathrm{~cm}$. In [17], were also approximately determined the first natural frequencies of the pipe $(170 \mathrm{~Hz}, 220 \mathrm{~Hz}, 260 \mathrm{~Hz})$ to check they were sufficiently away from the excitation frequency of $150 \mathrm{~Hz}$. The different behaviour of the response in the two tests can be attributed to the longer distances involved in the test but also on the different boundary conditions of the two pipes (the laboratory configuration and the present one).

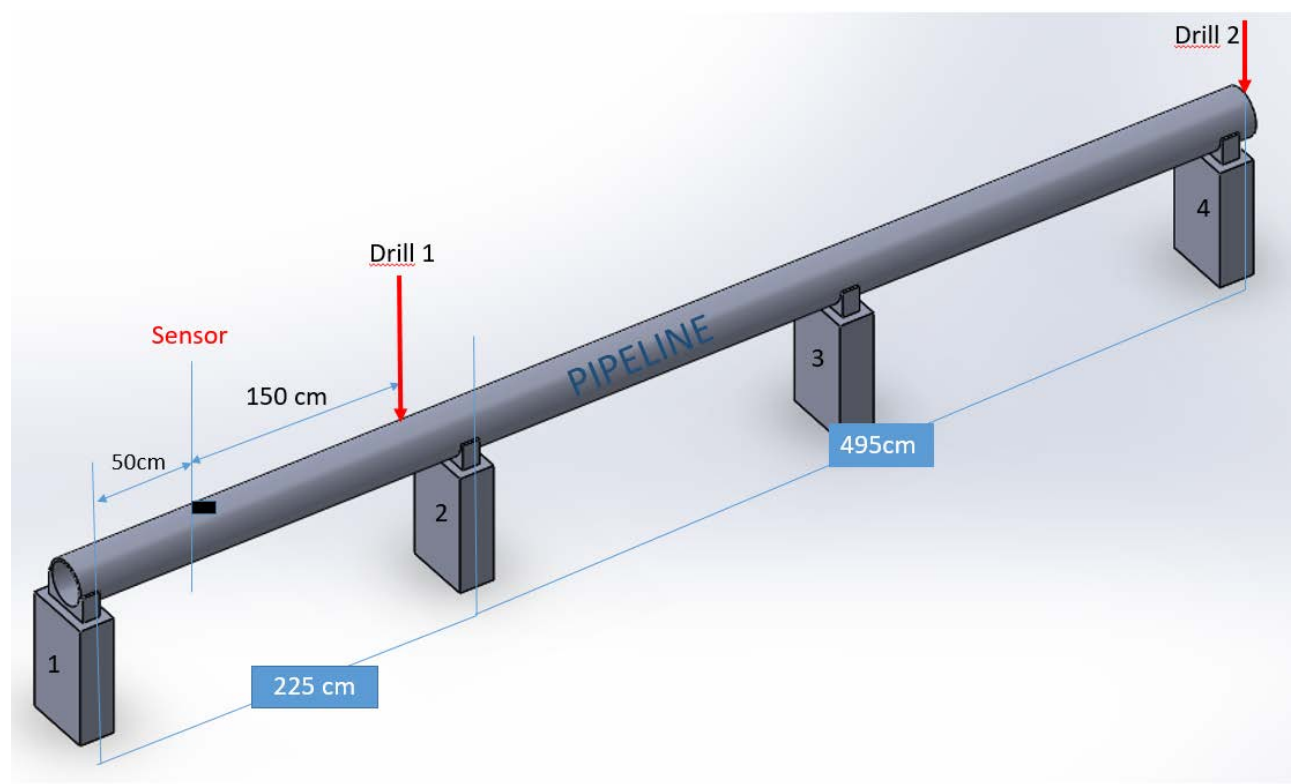

Figure 3: Scheme of the pipeline used in the outdoor test. 


\subsection{Tests on a high-pressure pipe under operations}

The vibration sensor, along with other kind of FBG sensors, has been tested at SITEC (Sustainable Innovation Technology) laboratory based in Casaccia Research Centre of ENEA [18]. The laboratory is equipped with two seismic tables for tests on structures up to 10 tons mass and $3 \mathrm{~g}$ acceleration. Three sensors have been applied on the pipe that carries the pressurized fluid to feed the hydraulic actuators of the tables: a vibration sensor, a copper coated FBG sensor and a bare FBG sensor. The copper coated one and the bare have been applied using glue to the pipe; the vibration sensor has been applied with a different system (under patent). The pressure inside the pipe is $50 \mathrm{~atm}$ when is not working and reaches 200 atm during seismic tests; the temperature of the fluid reaches $30-35^{\circ} \mathrm{C}$ to a maximum of $40^{\circ} \mathrm{C}$. A typical seismic test has been performed on the vibration table divided in three phases: in the first phase, the structure vibrates with low level excitation along each axis in order to acquire the resonance frequency; the second phase simulates an earthquake; in the third phase, the structure vibrates another time with low level of vibrations.

The results showed that the vibration sensor identify frequencies from $70 \mathrm{~Hz}$ to $150 \mathrm{~Hz}$, but is not usable to amplify lower and higher frequencies. The lower and higher frequencies were recorder by the bare FBG sensor attached to the pipe. As for the last FBG sensor, the copper coating did not improve its sensitivity toward vibrations when compared to the bare sensor.

\subsection{Optimization of the vibration sensor}

By exploiting the dynamical characteristics of the mount of the FBG in the SPFD sensor it is possible to amplify the response of the sensor itself. Here we summarize the results obtained in Ref. [19]. In the enforced motion, the amplification factor start from zero, at zero frequency base motion:

$$
\frac{Z_{r}}{Z_{t}}=\frac{\Omega^{2}}{\sqrt{\left(1-\Omega^{2}\right)^{2}+(2 \zeta \Omega)^{2}}} .
$$

The present mount of the sensor is not optimized and provides an amplification of 0.003715 [19]. Such a small value is due to the fact that $\Omega^{2}$ is very small for the FBG mount considered: $(150 \mathrm{~Hz} / 2577 \mathrm{~Hz})^{2}=0.00339$. If we can reduce the natural frequency of the mount to about $150 \mathrm{~Hz}$ we can reach an amplification factor in the range of 16.6-48 for damping factors in the range of $\zeta=0.03-0.01$ respectively. Of course, the smaller value of the damping corresponds to the higher values of the amplification factor. If we "tune" the sensor at the expected excitation frequency it will have an acceptable response in a wide range of frequencies. In fact, considering that with an amplification of only 0.0037 the sensor was able to detect the drilling operation few meters away we can, taking that value of amplification as a lower limit, estimate the bandwidth of operation of the SPFD sensor. With this sensor tuned at $150 \mathrm{~Hz}$ we can get an amplification higher than 0.0036 at frequencies above $9 \mathrm{~Hz}$. To obtain this result we shall consider that we need to increase the mass of the SPFD with $25 \mathrm{~g}$ of non-structural mass. By further increasing the value of the mass we can further increase the bandwidth of the sensor. If we need to increase the amplification even further that maybe possible with a proper design of the mounting considered as a multi-degree-of-freedom system. 


\subsection{Principle of management of sensor networks and satellite transmissions}

One of the advantages in using this kind of sensors is that the installer could create a long chain of them, multiplexing the various sensors in fewer lines than with conventional sensors. In order to control long pipelines, even thousands of $\mathrm{km}$, it is necessary to find a specific length in which the sensors have a good response without any loss of signal, or maintaining a good sensitivity. From the second test mentioned above, we can estimate a sensitivity of about 10 meters but according to the theory, shortly mentioned above, an improvement of 1 or 2 order of magnitude can be achieved in a fairly large excitation frequency bandwidth. This aspect will be assessed experimentally in a future work. An improved sensitivity will largely reduce the number of sensors required on a pipeline making much less expensive the installation and the data management.

Another advantage of the FBG and FBG based sensors is that they do not need recalibration so that they are particularly attractive for large infrastructure monitoring such as pipelines that need to be monitored for many years continuously. Furthermore, differently from electrical sensors, signals from FBGs can be detected even if the if the optical fibre is cut. That is possible because the signal is lost only in one direction, while the other direction is still working, so that the sensor signal can be collected anyway. For very long pipelines one can think of collecting the data on stations located few $\mathrm{km}$ or few tens of $\mathrm{km}$ away one another. All the data can be transformed in electronic format and sent to a central unit through conventional telecommunication channels or in case of remotely located pipelines through satellite transmission (Fig. 4).

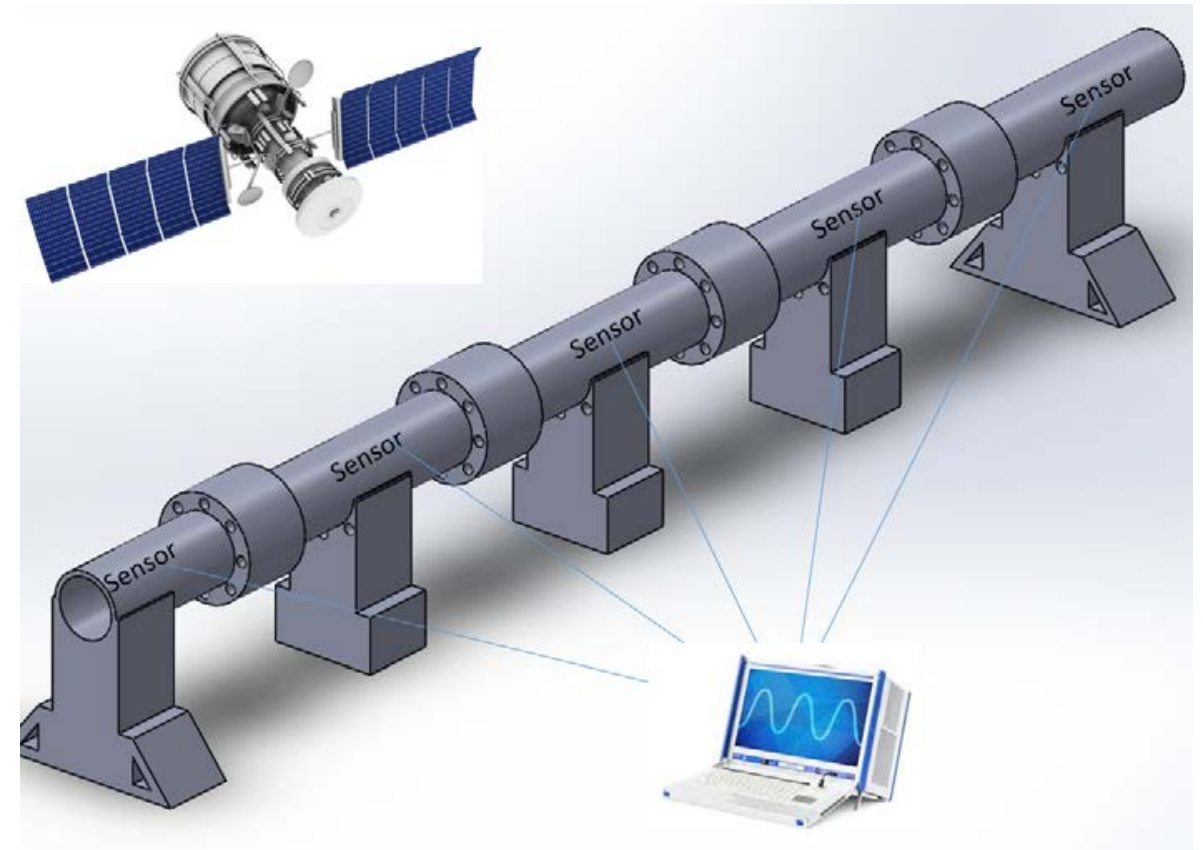

Figure 4: Sensor network and telecommunication with central control unit. 


\section{CONCLUSION}

An FBG based sensor for vibration monitoring on pipelines has been presented along with a review of the main results obtained. An improvement of the sensor sensitivity is outlined based on a single-degree-of-freedom spring mass model. A multi-degree-of-freedom model could show that a further improvement in the sensitivity in a larger bandwidth is possible. The theoretical consideration reported will be verified experimentally in a future work.

\section{ACKNOWLEDGEMENTS}

The authors acknowledge Sapienza University of Rome for grant 2015 "Sviluppo di smart structures ai fini della mitigazione degli effetti dei disastri ambientali (SDM, Smart Disaster Mitigation)" and the School of Aerospace Engineering for testing the decommissioned pipeline. A. Paolozzi and C. Paris acknowledge the Centro Fermi - Museo Storico della Fisica e Centro Studi e Ricerche "Enrico Fermi".

\section{REFERENCES}

[1] Oil pipeline explosion kills 105 in Nigeria; Institute for the Analysis of Global Security. Energy security, IAGS website, 26 June 2003, http://www.iags.org/ es062603. html\#nigeria. Accessed on: 15 Feb. 2016.

[2] Discovery of illegal pipeline taps double in Mexico as thieve drill into oil, gas ducts, Stevenson M., Phys.org website, Jul. 23, 2013, http://phys.org/news/2013-07-illegalpipeline-mexico.html. Accessed on: 20 May 2016.

[3] Theft of oil pipeline: ADR, spill outside airport customs fence, Aeroporti di Roma, 12 February 2015, http://tinyurl.com/jo4bedw. Accessed on: 15 Feb. 2016.

[4] Abbrescia, M. et al., The gas monitoring system for the Resistive Plate Chamber detector of the CMS experiment at LHC. Nuclear Physics B - Proceedings Supplements, 177, pp. 293-296, 2008.

[5] Benussi, L. et al., The Omega-like: a novel device using FBG sensors to position vertex detectors with micrometric precision. Nuclear Physics B - Proceedings Supplements, 172, pp. 263-265, 2007.

[6] Benussi, L. et al., Use of Fiber Bragg Grating Sensor for position monitoring in high energy physics experiment BTeV. Proceedings of IEEE Sensors, pp. 874-879, 2002.

[7] Benussi, L. et al., Study of gas purifiers for the CMS RPC detector. Nuclear Instruments and Methods in Physics Research, Section A: Accelerators, Spectrometers, Detectors and Associated Equipment, 661, pp. 241-244, 2012.

[8] Lupi, C., Felli, F., Ippoliti, L., Caponero, M.A., Fabbri, F. \& Paolozzi, A., Lead coating to improve fiber bragg grating sensitivity at cryogenic temperatures. Proceedings of SPIE - The International Society for Optical Engineering, 5855(2), pp. 811-815, 2005.

[9] Lupi, C., Felli, F., Caponero, M.A. \& Paolozzi, A., The effectiveness of metal coating on FBG sensor sensitivity at cryogenic temperature. Proceedings of SPIE - The International Society for Optical Engineering, 6619, pp. 161-164, 2007.

[10] Paolozzi, A., Caponero, M.A., Cassese, F. \& Leonardi, M., Use of embedded optical fibers for structural analysis. Proceedings of the XVII International Modal Analysis Conference - IMAC, 1, pp. 699-704, 1999.

[11] Saviano, G., Felli, F., Paolozzi, A., Caponero, M.A. \& Pugliese, L., Monitoring temperature and water imbibition in litic materials by embedded FBG. Proceedings of the 3rd European Workshop - Structural Health Monitoring, 2006

[12] Caponero, M.A., Felli, F., Paolozzi, A. \& Peroni, I., Vibration tests on metal alloys with embedded optical fibers. Proceedings of SPIE - The International Society for Optical Engineering, 4234, pp. 152-159, 2001. 
[13] Felli, F., Paolozzi, A. \& Caponero, M.A., Fabrication of Intelligent Aluminum Matrix Composite. Aluminum Transactions, 2, pp. 189-194, 2000.

[14] Cusano, A., Capoluongo, P., Campopiano, S., Cutolo, A., Giordano, M., Caponero, M., Felli, F. \& Paolozzi, A., Dynamic measurements on a star tracker prototype of AMS using fiber optic sensors, Smart Materials and Structures, 15, pp. 441-450, 2006

[15] Felli, F., Paolozzi A., Vendittozzi, C. \& Paris, C., Smart disaster mitigation in Italy. A brief overview on the state of the art. Proceedings of the ASME Conference on Smart Materials, Adaptive Structures and Intelligent Systems, SMASIS 2014, Paper No. SMASIS2014-7631, 2014.

[16] Felli, F., Paolozzi A., Vendittozzi, C., Paris, C., Asanuma, H., De Canio, G., Mongelli, M. \& Colucci, A., Structural health monitoring of pipelines for environment pollution mitigation. Proceedings of the ASME Conference on Smart Materials, Adaptive Structures and Intelligent Systems SMASIS 2015, Paper No. SMASIS2015-8922, 2015.

[17] Felli, F., Paolozzi, A., Vendittozzi, C., Paris, C. \& Asanuma, H., Analysis of FBG Sensors Data for Pipeline Monitoring. Proceedings of the ASME Conference on Smart Materials, Adaptive Structures and Intelligent Systems SMASIS 2016, Paper No. SMASIS2016-9260, 2016.

[18] Paris, C., Felli, F., Vendittozzi, C., Paolozzi, A., Mongelli, M., De Canio, G., Colucci, A. \& Asanuma, H., Test of FBG sensors for monitoring high pressure pipes, Proceedings of SPIE - The International Society for Optical Engineering, 10168, paper 101681Q, 2017.

[19] Paolozzi, A., Felli, F., Vendittozzi, C., Paris, C. \& Asanuma, H., Optimization of FBG sensor sensitivity for disaster prevention in pipelines. Proceedings of the ASME 2017 International Conference on Materials and Processing, ICM\&P, Paper No. ICMP2017-4445, 2017. 